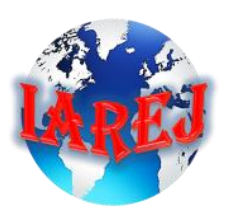

Research Article

\title{
Effect of aperture averaging on four petal Gaussian beams in atmospheric turbulence \\ Mert Bayraktar ${ }^{a} *$ (iD \\ ${ }^{a}$ Turkish Aerospace Industries, Havacılık Bulvarı No:17, Ankara, Turkey
}

\begin{tabular}{|c|c|}
\hline ARTICLE INFO & ABSTRACT \\
\hline $\begin{array}{l}\text { Article history: } \\
\text { Received 01 October } 2020 \\
\text { Revised } 06 \text { February } 2021 \\
\text { Accepted } 06 \text { March } 2021 \\
\text { Keywords: } \\
\text { Aperture averaging } \\
\text { Atmospheric turbulence } \\
\text { Four petal Gaussian beam } \\
\text { Scintillation }\end{array}$ & $\begin{array}{l}\text { Aperture averaged scintillation of four petal Gaussian beam is studied in this article. Split step } \\
\text { propagation approach which is used in wave propagation applications is selected to model } \\
\text { atmospheric turbulence. Results are plotted in two types. First type is the analysis of aperture } \\
\text { averaged scintillation versus propagation distance for constant receiver aperture. Second ones } \\
\text { involve scintillation performance applying aperture averaging at constant distance. All results are } \\
\text { compared with Gauss beam since commercial lasers generally radiates in Gaussian distribution. } \\
\text { We observe that four petal Gaussian beam becomes more advantageous under moderate turbulence } \\
\text { than weak one. In other point of view, it is possible to obtain less scintillation index by increasing } \\
\text { beam order. Our results are applicable optical applications operating in atmosphere. }\end{array}$ \\
\hline
\end{tabular}

(C) 2021, Advanced Researches and Engineering Journal (IAREJ) and the Author(s).

\section{Introduction}

Because of the recent developments in technology, communication systems with low latency and high data rate need to arise. Related with this, $5 \mathrm{G}$ and beyond technologies play a vital role to meet this demand. Free space optics systems are the one of the components of $5 \mathrm{G}$ systems. Free space optics systems provide low latency and higher bit rate but performance of these systems is dependent on the atmospheric conditions. One way to overcome the negative effects of atmosphere is beam shaping. Utilizing nonconventional beams, performance of free space optics systems can be improved.

Propagation of different types of beams through random media attracts the attentions of scientists in different points of views. Intensity profiles, scintillation behavior, beam size, and coherency are some of these points [1]. Wave propagation lies in the background of this analysis. Solution of Huygens-Fresnel integral gives the received field through free space or turbulence [2]. For complex source field expressions, split step propagation methods are also used [3]. In some studies, accuracy of this method is also increased [4]. Benefiting from these methods scintillation index which is the most effective noise factor
[5] is measured for untraditional beams. While scintillation index of sine hollow beam is less than Gauss beam [6], less scintillation index than sine hollow beam can be provided by Mathieu-Gauss beam [7]. In addition, we prove that cylindrical-sinc Gaussian beam has less scintillation index than Gauss beam [8]. Besides this this type of beam has a diverging nature propagating in atmospheric turbulence [9]. Considering the effect of scintillation, authors show how to detect information using Gaussian vortex beam [10]. In addition to above beams, there are some other types of beams that provides less scintillation. Regarding with this, it is shown in [11,12] that Airy and partially coherent Airy beams have less scintillation than Gauss beam. Similarly, scintillation index of truncated flat-topped beam is low [13]. Poynting vector of Weber beam is calculated in [14]. For high frequency wave propagation, the Eulerian Gaussian beam method is generalized in [15].

In addition to methods and some applications listed above, four petal Gaussian beam is introduced by propagating through $\mathrm{ABCD}$ system [16]. If Vortex is added to four petal Gauss beam, it is observed that topological charge is quite dominant on the central hollow in far field [17]. While four petal Gaussian vortex beam

* Corresponding author. Tel.: +90-312-811-1800; Fax: +90-312-811-1425

E-mail addresses: mert.bayraktar@tai.com.tr (M. Bayraktar)

ORCID: 0000-0002-0337-7650 (M. Bayraktar)

DOI: $10.35860 /$ iarej. 803508

This article is licensed under the CC BY-NC 4.0 International License (https://creativecommons.org/licenses/by-nc/4.0/). 
shows clockwise phase distribution through the propagation in uniaxial crystal [18], four petal LorentzGauss beam turns into elliptical Gaussian beam in the same medium [19]. $M^{2}$, propagation factor for paraxial region is derived in [20]. Four petal Gaussian beam is propagated through fractional Fourier system and it is concluded as fraction, aperture, and source parameters are effective in received field [21]. Besides these, free space propagation and propagation in turbulent medium is also another interesting field for wave propagation. In the light of this, raise in topological charge provides larger hollow in the center for four petal Lorentz-Gauss vortex beam [22]. Similarly, larger topological charge reduces the effect of atmospheric turbulence on partially coherent elliptic Gaussian vortex beams [23]. Partially coherent four petal Gaussian beam shows resistance to oceanic turbulence if it is generated with larger beam order [24]. Finally, it is shown in [25] that four petal Gaussian vortex beam having incoherency evolves into Gaussian like shape easier if strength of turbulence increases. Airy transform is applied to four petal Gaussian beam and Airy like beam is obtained at the output [26].

Bearing in mind above literature review, we study the aperture averaged scintillation for four petal Gaussian beam under weak and moderate atmospheric turbulent regime. We use split step propagation to model the atmosphere. This model is used for propagate wave through turbulent medium. We analyzed the results with respect to propagation distance and size of receiver aperture. We anticipate that our results will be useful for optical communication and range measurement systems' designers.

\section{Source Field Distribution and Method to Measure Performance}

\subsection{Four petal gaussian beam}

Source field expression is written for four petal Gaussian beam [16] as

$$
u_{s}\left(s_{x}, s_{y}\right)=\left(\frac{s_{x} s_{y}}{\alpha^{2}}\right)^{2 n} \exp \left(-\frac{s_{x}^{2}+s_{y}^{2}}{\alpha^{2}}\right)
$$

where $n=0,1,2,3 \ldots$. refer to beam order, $s_{x}, s_{y}$ are transverse plane source coordinates, and $\alpha=\sqrt{\alpha_{x}^{2}+\alpha_{y}^{2}}$ being the Gaussian source size. Based on equation.1, Figure 1 and 2 are plotted to show the effect of beam order and Gaussian source size on the initial plane. It is seen from Figure 1 that while beam order increases, hollow region in the center gets larger. When beam order is set to 1, effect of Gaussian source size is seen in Figure 2. For asymmetric case $\alpha_{x}>\alpha_{y}$, beam lies along $\mathrm{x}$-axis. Similar idea is valid for $\alpha_{y}>\alpha_{x}$. Furthermore, beam spreads on transverse plane symmetrically if $\alpha_{x}, \alpha_{y}$ are increased in the same amount.

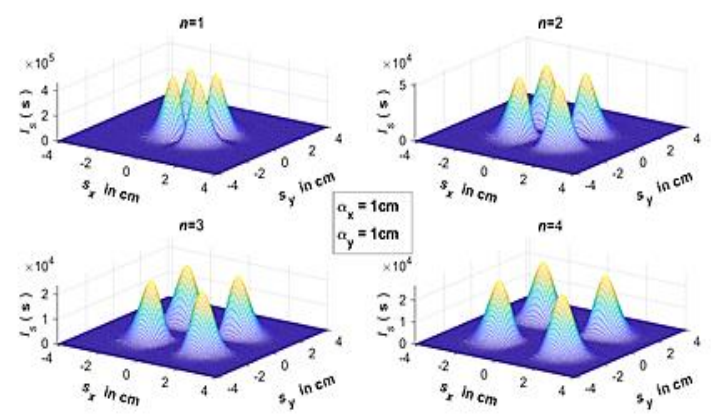

Figure 1. Transverse source plane intensity distribution for constant Gaussian source size

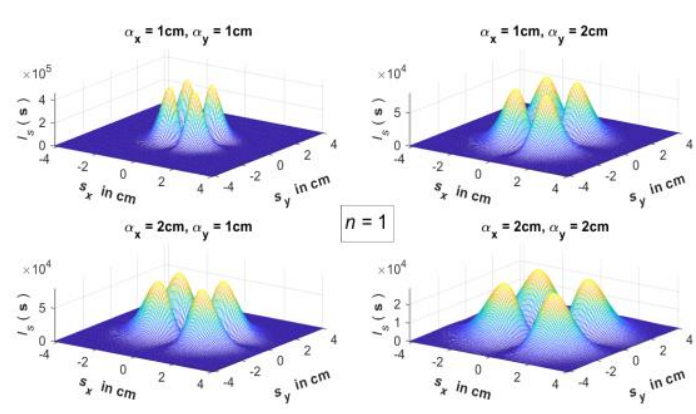

Figure 2. Transverse source plane intensity distribution for constant beam order

\subsection{Measurement of scintillation in atmosphere}

Numerical split step propagation set-up involves special values presented in table 1 below. In this set-up, between the screens, free space conditions are satisfied. We chose modified von-Karman power spectral density since it is the closest model to Hill spectrum. Refractive index structure constant is the parameter to determine the strength of turbulence and it is taken into account in $r_{0}$. To satisfy the averaging in Eq. 4, number of realizations is taken as 500 which corresponds to infinity in reality. To avoid the complexity of computation of Huygens-Fresnel integral, this method benefits from convolution property of Fourier transform as it is seen from Eq. 2.

After applying all settings, received field after one step is found as

$$
u_{r}\left(r_{x}, r_{y}, L\right)=A \mathrm{xF}^{-1}\left(\begin{array}{l}
F\left[\left(\frac{s_{x} s_{y}}{\alpha^{2}}\right)^{2 n} \exp \left(-\frac{s_{x}^{2}+s_{y}^{2}}{\alpha^{2}}\right)\right] \\
F\left\{\exp \left[\frac{j k}{2 L}\left(s_{x}^{2}+s_{y}^{2}\right)\right]\right\}
\end{array}\right)
$$

where

$$
A=\frac{-j k \exp (j k L)}{2 \pi L} \exp \left[\frac{j k}{2 L}\left(r_{x}^{2}+r_{y}^{2}\right)\right] \exp [\psi(r)]
$$

here $k$ denotes wave number, $r_{x}, r_{y}$ are receiver plan coordinates, $F$ and $F^{-1}$ refer to Fourier and inverse Fourier transform, and $\psi(r)$ indicates phase fluctuations due to atmosphere and it involves power spectral density. Then, aperture averaged scintillation is evaluated benefiting from received field as: 


$$
m^{2}=\frac{\left\langle P\left(r_{x}, r_{y}\right)^{2}\right\rangle}{\left\langle P\left(r_{x}, r_{y}\right)\right\rangle^{2}}-1=\frac{\left(\int u_{r}\left(r_{x}, r_{y}\right) u_{r}^{*}\left(r_{x}, r_{y}\right) d S\right)^{2} / N}{\left(\int u_{r}\left(r_{x}, r_{y}\right) u_{r}{ }^{*}\left(r_{x}, r_{y}\right) d S / N\right)^{2}}
$$

where * indicates complex conjugate. Adapted model of above mathematical equations is presented in Figure 3.

\section{Results and Discussions}

In this part of the study, we comment on the numerical results.

Table 1. Numerical set-up parameters

\begin{tabular}{|c|c|}
\hline $\begin{array}{l}\text { Source plane } \\
\text { dimensions }(S)\end{array}$ & $10 \mathrm{~cm} \mathrm{X} \mathrm{10cm}$ \\
\hline $\begin{array}{l}\text { Propagation } \\
\text { distance }(L)\end{array}$ & Up to $5500 \mathrm{~m}$ \\
\hline $\begin{array}{l}\text { Power spectral } \\
\text { density }\end{array}$ & $\phi=0.023 r_{0}^{-\frac{5}{3}} \frac{\exp \left(-\left(\frac{f}{f_{m}}\right)^{2}\right)}{\left(f^{2}+f_{0}^{2}\right)^{\frac{11}{6}}}$ \\
\hline $\begin{array}{l}\text { Refractive index } \\
\text { structure constant } \\
\left(C_{n}^{2}\right)\end{array}$ & $\begin{array}{l}\text { For weak turbulence: } 10^{-14} \mathrm{~m}^{-\frac{2}{3}} \\
\text { For moderate turbulence : } \\
10^{-13} \mathrm{~m}^{-\frac{2}{3}}\end{array}$ \\
\hline $\begin{array}{l}\text { Grid size in transverse } \\
\text { plane }\end{array}$ & $512 \times 512$ \\
\hline $\begin{array}{l}\text { Inner scale frequency } \\
\left(f_{m}\right)\end{array}$ & $\frac{5.92}{l_{0} 2 \pi}, l_{0} \rightarrow 0$ \\
\hline $\begin{array}{l}\text { Outer scale frequency } \\
\left(f_{0}\right)\end{array}$ & $\frac{1}{L_{0}}, \quad L_{0} \rightarrow \infty$ \\
\hline Fried parameter & $r_{0}=\left(0.423\left(\frac{2 \pi}{\lambda}\right)^{2} C_{n}^{2} L\right)^{-\frac{3}{5}}$ \\
\hline $\begin{array}{l}\text { Operating wavelength } \\
(\lambda)\end{array}$ & $1550 \mathrm{~nm}$ \\
\hline Number of screens & $\begin{array}{l}\text { 21: } 0-1200 \mathrm{~m} \\
51: 1200-3200 \mathrm{~m} \\
91: 3200-5500 \mathrm{~m}\end{array}$ \\
\hline $\begin{array}{l}\text { Receiver plane } \\
\text { dimensions in Figures } \\
4 \text { and } 5\end{array}$ & $25 \times 25$ grids \\
\hline $\begin{array}{l}\text { Propagation distance } \\
\text { in Figures } 6 \text { and } 7\end{array}$ & $3300 \mathrm{~m}$ \\
\hline $\begin{array}{l}\text { Realization amount } \\
\text { for averaging }(N)\end{array}$ & 500 \\
\hline
\end{tabular}

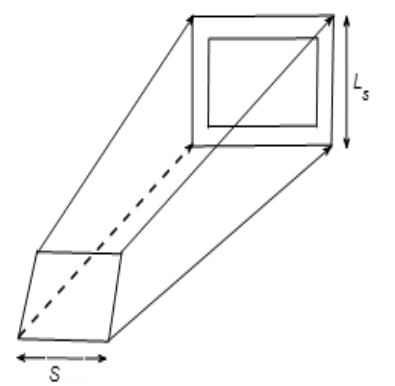

Figure 3. Propagation model through the atmosphere

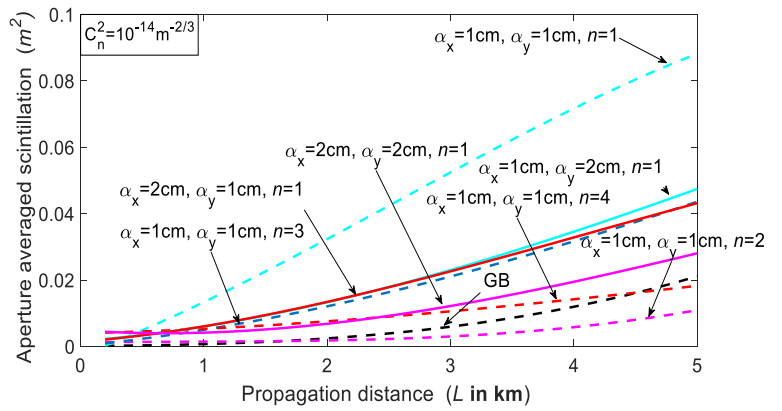

Figure 4. Aperture averaged scintillation of selected beam versus propagation path under weak turbulence

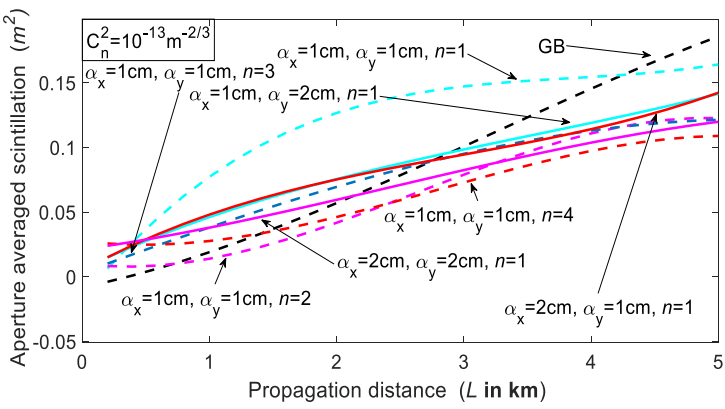

Figure 5. Aperture averaged scintillation of selected beam versus propagation path under moderate turbulence

We show aperture averaged scintillation index variations against propagation distance in Figures 4 and 5. It is seen from these figures that while only second order symmetric beam has advantageous as compared to Gauss beam in weak turbulence, all settings of four petal Gaussian beams have lower scintillation index for stronger turbulence regime.

In moderate turbulence case, scintillation index decreases as beam order raises. Additionally, increase in source size provides similar result with larger beam order. Besides this, asymmetry, without considering the direction, brings scintillation reduction.

In order to show the advance of four petal Gaussian beam, Table 2 is given. As it is observed from this table, however scintillation index of fourth order Four petal Gaussian beam is higher than Gaussian beam at close distance, significant amount of scintillation reduction can be provided by Four petal Gaussian beam at longer distances. At $5 \mathrm{~km}$, scintillation of Gaussian beam is approximately two times higher than Four petal Gaussian beam.

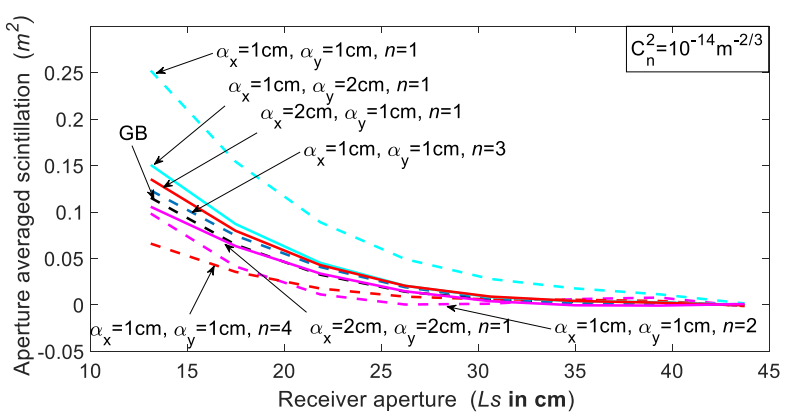

Figure 6. Aperture averaged scintillation of selected beam versus receiver aperture under weak turbulence. 
Table 2. Aperture averaged scintillation index under moderate turbulence.

\begin{tabular}{|c|c|c|}
\hline Distance $(\mathrm{km})$ & Gauss beam & $\begin{array}{c}\text { Fourth order } \\
\text { Four petal } \\
\text { Gaussian beam }\end{array}$ \\
\hline 0.4 & 0.001086 & 0.02483 \\
\hline 0.6 & 0.006571 & 0.02489 \\
\hline 0.8 & 0.01256 & 0.02586 \\
\hline 1 & 0.01903 & 0.02767 \\
\hline 1.2 & 0.02592 & 0.03022 \\
\hline 1.4 & 0.03321 & 0.03343 \\
\hline 1.6 & 0.04084 & 0.03723 \\
\hline 1.8 & 0.04879 & 0.04151 \\
\hline 2 & 0.05071 & 0.04621 \\
\hline 2.6 & 0.0829 & 0.06194 \\
\hline 3 & 0.1008 & 0.07293 \\
\hline 3.6 & 0.1278 & 0.08853 \\
\hline 4 & 0.1454 & 0.09734 \\
\hline 5 & 0.1859 & 0.109 \\
\hline
\end{tabular}

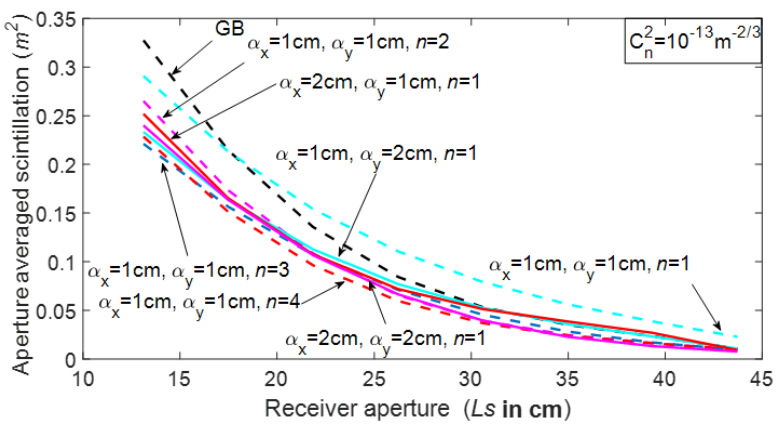

Figure 7. Aperture averaged scintillation of selected beam versus propagation receiver aperture under moderate turbulence

In other point of view, four petal Gaussian beam has lesser scintillation index with versus receiver aperture opening as it is seen from Figures 6 and 7 . We see that second and fourth order beam has lesser scintillation index for small receiver apertures. In addition, large source size four petal Gaussian beam has similar performance with Gauss beam under weak turbulence conditions. First order beam has the largest scintillation index. Then asymmetric ones and third order beams follow it.

As it is wanted, scintillation mitigation is seen for moderate turbulence as it is shown in Figure 7. We see that scintillation index of all kinds of four petal beams is less than Gauss beam for small apertures. In advantageous region, from the lowest scintillation index up to the highest one beams are listed as third, fourth order beams, asymmetric and large source size beams, second, and first order beams. We can investigate that four petal beam has less point like scintillation index as compared to Gauss beam since it can be evaluated in small aperture openings. This advantage vanishes when aperture size gets wider.

\section{Conclusion}

Aperture averaged scintillation of four petal Gaussian beam is studied in this article. Numerical results show that beam order and scintillation index is inversely proportional to each other. Additionally, scintillation index of four petal Gaussian beam is quite advantageous as compared to Gaussian beam for small aperture openings under moderate turbulence. In addition, four petal Gaussian beam is resistive to atmospheric turbulence. Because, scintillation index of all selected types four petal Gaussian beam is becomes advantageous when turbulence strength raises. Consequently, in the light of these investigations, four petal Gaussian beam can be selected as source beam for optical wireless communication and LIDAR systems operating in turbulence. In the future, our studies will focus on to measure the scintillation of other beams and generation of four petal beam experimentally.

\section{Declaration}

The author declared no potential conflicts of interest with respect to the research, authorship, and/or publication of this article. The author also declared that this article is original, was prepared in accordance with international publication and research ethics, and ethical committee permission or any special permission is not required.

\section{Author Contributions}

M. Bayraktar is responsible for all parts of the study.

\section{Nomenclature}

\begin{tabular}{|c|c|c|}
\hline$\alpha=\sqrt{\alpha_{x}^{2}+\alpha_{y}^{2}}$ & : & Gaussian source size (m) \\
\hline$C_{n}^{2}$ & - & $\begin{array}{l}\text { Refractive } \\
\text { constant }\end{array}$ \\
\hline$f_{\mathrm{m}}$ & : & Inner scale frequency \\
\hline$f_{\mathrm{o}}$ & : & Outer scale frequency \\
\hline$F$ & : & Fourier transform \\
\hline$F^{-1}$ & : & Inverse Fourier transform \\
\hline$k$ & : & Wave number $\left(m^{-1}\right)$ \\
\hline$L$ & : & Propagation distance \\
\hline$L_{s}$ & : & Receiver plane size \\
\hline$m^{2}$ & : & $\begin{array}{l}\text { Aperture averaged scintillation } \\
\text { index }\end{array}$ \\
\hline$n$ & : & beam order $(0,1,2 \ldots)$ \\
\hline$r_{x}, r_{y}$ & : & Receiver plane coordinates(m) \\
\hline$S_{x}, S_{y}$ & : & Source plane coordinates $(\mathrm{m})$ \\
\hline$S$ & : & Source plane size \\
\hline$u_{s}$ & : & Source field expression \\
\hline$u_{r}$ & : & Received field expression \\
\hline$\psi(r)$ & • & Phase fluctuations \\
\hline$\lambda$ & : & Operating wavelength \\
\hline
\end{tabular}

\section{References}

1. Wenzel, A.R., Localization of the Mean and Mean Squared Intensities, and Intensity Fluctuations, of Waves 
Propagating in a One-Dimensional Random Medium. Wave Motion, 1985. 7(6): p. 589-600.

2. Andrews, L.C., Laser Beam Propagation Through Random Media. 2 ed. 2005, Washington: SPIE.

3. Schmidt, J.D., Numerical Simulation Optical Wave Propagation with examples in MATLAB. 2010: SPIE.

4. de Hoop, M.V., J.H. Le Rousseau, and R.S. Wu, Generalization of the phase-screen approximation for the scattering of acoustic waves. Wave Motion, 2000. 31(1): p. 43-70.

5. Eyyuboglu, H.T. and M. Bayraktar, SNR bounds of FSO links and its evaluation for selected beams. Journal of Modern Optics, 2015. 62(16): p. 1316-1322.

6. Bayraktar, M., Estimation of scintillation and bit error rate performance of sine hollow beam via random phase screen. Optik, 2019. 188: p. 147-154.

7. Bayraktar, M., Scintillation and bit error rate calculation of Mathieu-Gauss beam in turbulence. Journal of Ambient Intelligence and Humanized Computing, 2020.

8. Bayraktar, M., Scintillation and bit error rate analysis of cylindrical-sinc Gaussian beam. Physica Scripta, 2020. 95(11).

9. Eyyuboglu, H.T. and M. Bayraktar, Propagation properties of cylindrical sinc Gaussian beam. Journal of Modern Optics, 2016. 63(17): p. 1706-1712.

10. Eyyuboglu, H.T., Optical communication system using Gaussian vortex beams. Journal of the Optical Society of America a-Optics Image Science and Vision, 2020. 37(10): p. 1531-1538.

11. Eyyuboglu, H.T. and E. Sermutlu, Partially coherent Airy beam and its propagation in turbulent media. Applied Physics B-Lasers and Optics, 2013. 110(4): p. 451-457.

12. Eyyuboglu, H.T., Scintillation behavior of Airy beam. Optics and Laser Technology, 2013. 47: p. 232-236.

13. Liu, X.L., et al., Scintillation properties of a truncated flattopped beam in a weakly turbulent atmosphere. Optics and Laser Technology, 2013. 45: p. 587-592.

14. Rondon-Ojeda, I. and F. Soto-Eguibar, Properties of the Poynting vector for invariant beams: Negative propagation in Weber beams. Wave Motion, 2018. 78: p. 176-184.

15. $\mathrm{Wu}, \mathrm{H}$. and X. Yang, The Eulerian Gaussian beam method for high frequency wave propagation in the reduced momentum space. Wave Motion, 2013. 50(6): p. 1036-1049.

16. Duan, K.L. and B.D. Lu, Four-petal Gaussian beams and their propagation. Optics Communications, 2006. 261(2): p. 327-331.

17. Guo, L.N., Z.L. Tang, and W. Wan, Propagation of a fourpetal Gaussian vortex beam through a paraxial $A B C D$ optical system. Optik, 2014. 125(19): p. 5542-5545.

18. Liu, D.J., et al., Evolution properties of four-petal Gaussian vortex beam propagating in uniaxial crystals orthogonal to the optical axis. European Physical Journal D, 2015. 69(9).

19. Liu, D.J., et al., Properties of a four-petal Lorentz-Gauss beam propagating in uniaxial crystal orthogonal to the optical axis. Optik, 2019. 183: p. 257-265.

20. Zhou, G.Q. and Y. Fan, M-2 factor of four-petal Gaussian beam. Chinese Physics B, 2008. 17(10): p. 3708-3712.

21. Tang, B., Propagation of four-petal Gaussian beams in apertured fractional Fourier transforming systems. Journal of Modern Optics, 2009. 56(17): p. 1860-1867.

22. Deng, W.T., et al., Four-petal Lorentz-Gauss vortex beam and its propagation in free space. Optik, 2020. 202.
23. Wu, K.N., et al., Propagation of partially coherent fourpetal elliptic Gaussian vortex beams in atmospheric turbulence. Optics Express, 2018. 26(23): p. 30061-30075.

24. Liu, D.J., et al., Evolution properties of partially coherent four-petal Gaussian beams in oceanic turbulence. Journal of Modern Optics, 2017. 64(16): p. 1579-1587.

25. Liu, D.J., et al., Propagation properties of partially coherent four-petal Gaussian vortex beams in oceanic turbulence. Laser Physics, 2017. 27(1): 016001.

26. Yaalou, M., Z. Hricha, and A. Belafhal, Investigation on Airy transform of Four-Petal Gaussian beams. Optical and Quantum Electronics, 2020. 52: 165.

27. Bayraktar, M. and H.T. Eyyuboglu, Propagation properties of optical bottle beam in turbulence. Optical Engineering, 2019. 58(3): 036104. 